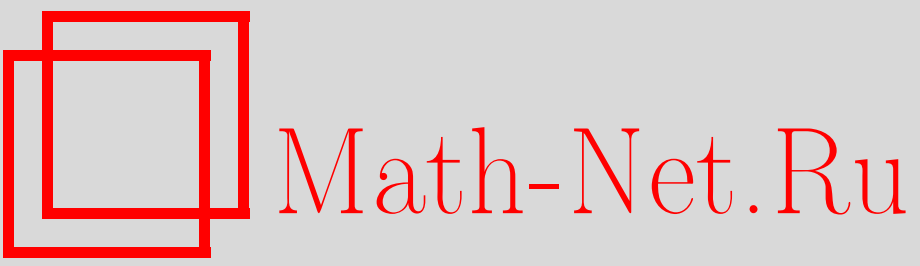

В. М. Долгополов, И. Н. Родионова, Задача с сопряжением на характеристической плоскости для одного пространственного аналога уравнения гиперболического типа, Вестн. Сам. гос. техн. ун-та. Сер. Физ.-мат. науки, 2010, выпуск 1(), 16-23

DOI: https://doi.org/10.14498/vsgtu759

Использование Общероссийского математического портала Math-Net.Ru подразумевает, что вы прочитали и согласны с пользовательским соглашением http: //www. mathnet.ru/rus/agreement

Параметры загрузки:

IP: 3.95 .254 .165

26 апреля 2023 г., $17: 05: 43$ 
УДК 811.93:004.912

\section{ЗАДАЧА С СОПРЯЖЕНИЕМ НА ХАРАКТЕРИСТИЧЕСКОЙ ПЛОСКОСТИ ДЛЯ ОДНОГО ПРОСТРАНСТВЕННОГО АНАЛОГА УРАВНЕНИЯ ГИПЕРБОЛИЧЕСКОГО ТИПА}

\section{В. М. Долгополов, И. Н. Родионова}

Самарский государственный университет, 443011, Самара, ул. ак. Павлова, 1

E-mail: mvdolg@ssu.samara.ru

Для одного пространственного аналога гиперболического уравнения в области, частями границы которой являются плоскости сингулярности коэбфициентов данного уравнения, решается краевая задача со склейкой на характеристической плоскости во введенном авторами специальном классе.

Ключевые слова: интегралъные уравнения, краевые задачи, уравнения гиперболического типа.

Введение. Настоящая работа является продолжением исследований, начатых в работах [1-3]. В работе рассмотрен пространственный аналог уравнения гиперболического типа в области, ограниченной двумя плоскостями сингулярности коэффициентов данного уравнения с условиями сопряжения на характеристической плоскости. Следует отметить, что при $\lambda=0$ подобная задача рассматривалась в работе [4], однако наличие слагаемого $\lambda U$ в уравнении вносит свои коррективы в решение задач со склейкой на характеристике, т. к. в этом случае задачи сводятся не к интегральным уравнениям Абеля $(\lambda=0)$, а к уравнениям с несвёрточными операторами, содержащими функцию ${ }_{0} F_{1}(a, z)$.

1. Пространственная задача MS. Рассмотрим уравнение

$$
U_{x y z}+\frac{2 p y}{(x-z)^{2}-y^{2}} U_{x z}-\frac{2 p(x-z)}{(x-z)^{2}-y^{2}} U_{y z}-4 \lambda \operatorname{sgn} y y(x-z) U_{z}=0 \quad\left(0<p<\frac{1}{2}\right)
$$

на множестве $\mathcal{G}=\mathcal{G}_{1} \cup \mathcal{G}_{2}$, где

$$
\begin{aligned}
& \mathcal{G}_{1}=\{(x, y, z): 0<z<x-y, 0<y<x<h\}, \\
& \mathcal{G}_{2}=\{(x, y, z): 0<z<x+y, 0<-y<x<h\}, \quad h>0 .
\end{aligned}
$$

Части границы $\mathcal{G}$, заданные уравнениями $z=x-y$ и $z=x+y$, являются плоскостями сингулярности коэффициентов уравнения (1).

ЗАДача MS. На множестве $\mathcal{G}$ найти решение уравнения (1), непрерывное в $\overline{\mathcal{G}}$, с данными

$$
\begin{gathered}
U(x, y, x-y)=\tau_{1}(x, y), \quad 0 \leqslant x \leqslant y \leqslant h, \\
U(x, y, x+y)=\tau_{2}(x, y), \quad 0 \leqslant x \leqslant-y \leqslant h,
\end{gathered}
$$

а также

$$
U(h, y, z)=\varphi_{1}(y, z), \quad(y, z) \in \bar{\Delta}_{3}=\{(y, z): 0 \leqslant y \leqslant h-z, 0 \leqslant z \leqslant h\},
$$

Вячеслав Михайлович Долгополов (к.ф.-м.н., доц.), доцент, каф. математики, информатики и математических методов в экономике. Ирина Николаевна Родионова (к.ф.-м.н., доц.), доцент, каф. математики, информатики и математических методов в экономике. 


$$
U(h, y, z)=\varphi_{2}(y, z), \quad(y, z) \in \bar{\Delta}_{3}^{*}=\{(y, z): 0 \leqslant-y \leqslant h-z, 0 \leqslant z \leqslant h\}
$$

и с условиями сопряжения на плоскости $y=0$ :

$$
\lim _{y \rightarrow 0-0}\left(U_{y}-U_{x}\right)=\lim _{y \rightarrow 0+0}\left(U_{y}+U_{x}\right) .
$$

От заданных функций потребуем выполнения следующих условий:

$$
\begin{gathered}
\left(\tau_{1}\right)_{x y} \in C\left(\bar{\Delta}_{1}\right), \quad\left(\tau_{2}\right)_{x y} \in C\left(\bar{\Delta}_{2}\right), \\
\tau_{1}(h, y)=\tau_{2}(h, y)=0, \quad \tau_{1}(x, 0)=\tau_{2}(x, 0), \\
\frac{\partial}{\partial x} \tau_{1}(x, 0)+\frac{\partial}{\partial y} \tau_{1}(x, 0)=\frac{\partial}{\partial y} \tau_{2}(x, 0)-\frac{\partial}{\partial x} \tau_{2}(x, 0), \\
\varphi_{1}(h-z, z)=\frac{\partial}{\partial z} \varphi_{1}(h-z, z)=\frac{\partial^{2} \varphi_{1}(h-z, z)}{\partial z \partial y}=0, \\
\varphi_{2}(z-h, z)=\frac{\partial}{\partial z} \varphi_{2}(z-h, z)=\frac{\partial^{2} \varphi_{2}(z-h, z)}{\partial z \partial y}=0 .
\end{gathered}
$$

Обозначим

$$
\begin{array}{cc}
\frac{\partial}{\partial y}\left[\frac{\partial^{2} \varphi_{1}(y, z)}{\partial z \partial y} \frac{1}{y}\right]=\Phi_{1}(y, z), & \frac{\partial}{\partial y}\left[\frac{\partial^{2} \varphi_{2}(-y, z)}{\partial z \partial y} \frac{1}{y}\right]=\Phi_{2}(y, z), \\
\Phi_{1}(y, z) \in C\left(\bar{\Delta}_{3}\right), & \Phi_{2}(y, z) \in C\left(\bar{\Delta}_{3}\right) .
\end{array}
$$

Пользуясь результатами работы [3], определим решение специального класса $R_{h}$ видоизмененной задачи Коши для уравнения (1) (задачи $\mathrm{C}$ ).

ОПределениЕ. Будем говорить, что классическое решение задачи $\mathrm{C}$ для уравнения $(1)$ в области $\mathcal{G}_{1}$ с данными $(2)$ и

$$
\begin{gathered}
\lim _{z \rightarrow x-y-0}(x-y-z)^{-2 p}(2 y)^{-2 p}\left(U_{x z}-U_{y z}\right)=\mu_{1}(x, y), \quad 0<x<y<h, \\
\lim _{z \rightarrow x-y-0} \frac{\partial U}{\partial z}=\nu_{1}(x, y), \quad 0<x<y<h
\end{gathered}
$$

принадлежат специальному классу $R_{h}$, если заданная функция $\nu_{1}$ имеет следующее интегральное представление:

$\nu_{1}(x, x-y)=\int_{x}^{h} T_{1}(\xi, x-y)\left[(\xi-x+y)^{2}-y^{2}\right]_{0}^{2 p} F_{1}\left(1+p ;-\lambda\left[(\xi-x+y)^{2}-y^{2}\right]^{2}\right) d \xi$,

где $T_{1}(x, y)$ - новая функция, непрерывная в области $\Delta_{1}=\{(x, y): 0<x<$ $<y<h\}$ и интегрируемая в $\bar{\Delta}_{1}$. Такие же условия налагаются и на функции $\nu_{1}(x, y)$ и $\mu_{1}(x, y),\left(\tau_{1}\right)_{x y} \in C\left(\bar{\Delta}_{1}\right) ;{ }_{0} F_{1}(a, z)=\sum_{n=0}^{\infty} \frac{z^{n}}{(a)_{n} n !}$.

Решение задачи $\mathrm{C}$ специального класса $R_{h}$ в области $\mathcal{G}_{1}$ имеет вид

$$
U(x, y, z)=\tau_{1}(x, y)-\int_{z}^{x-y} d s \int_{y+s}^{x} N_{1}(\xi, s)\left[(\xi-s)^{2}-y^{2}\right]^{p}\left[(x-s)^{2}-(\xi-s)^{2}\right]^{p} \times
$$




$$
\begin{gathered}
\quad \times{ }_{0} F_{1}\left(1+p ; \lambda\left[(\xi-s)^{2}-y^{2}\right]\left[(x-s)^{2}-(\xi-s)^{2}\right]\right) d \xi- \\
-\int_{z}^{x-y} d s \int_{x}^{h} T_{1}(\xi, s)\left[(\xi-s)^{2}-y^{2}\right]^{p}\left[(\xi-s)^{2}-(x-s)^{2}\right]^{p} \times \\
\quad \times{ }_{0} F_{1}\left(1+p ; \lambda\left[(\xi-s)^{2}-y^{2}\right]\left[(x-s)^{2}-(\xi-s)^{2}\right]\right) d \xi .
\end{gathered}
$$

Аналогично, решением задачи $\mathrm{C}$ в специальном классе $R_{h}$ в области $\mathcal{G}_{2}$ будет функция

$$
\begin{aligned}
U(x, y, z)= & \tau_{2}(x, y)-\int_{z}^{x+y} d s \int_{s-y}^{x} N_{2}(\xi, s)\left[(\xi-s)^{2}-y^{2}\right]^{p}\left[(x-s)^{2}-(\xi-s)^{2}\right]^{p} \times \\
& \times{ }_{0} F_{1}\left(1+p ; \lambda\left[(\xi-s)^{2}-y^{2}\right]\left[(x-s)^{2}-(\xi-s)^{2}\right]\right) d \xi- \\
- & \int_{z}^{x+y} d s \int_{x}^{h} T_{2}(\xi, s)\left[(\xi-s)^{2}-y^{2}\right]^{p}\left[(\xi-s)^{2}-(x-s)^{2}\right]^{p} \times \\
& \times{ }_{0} F_{1}\left(1+p ; \lambda\left[(\xi-s)^{2}-y^{2}\right]\left[(x-s)^{2}-(\xi-s)^{2}\right]\right) d \xi, \quad(15)
\end{aligned}
$$

удовлетворяющая в области $\mathcal{G}_{2}$ уравнению $(1)$, граничным условиям (3) и

$\lim _{z \rightarrow x+y-0} \frac{\partial U}{\partial z}=\nu_{2}(x, y), \quad \lim _{z \rightarrow x+y-0}(x+y-z)^{-2 p}(-2 y)^{-2 p}\left(U_{x z}+U_{y z}\right)=\mu_{2}(x, y)$,

где $\mu_{2}, \nu_{2}$ - заданные функции непрерывные в $\Delta_{2}=\{(x, y): 0<x<-y<h\}$ и интегрируемые в $\bar{\Delta}_{2} ;\left(\tau_{2}\right)_{x y} \in C\left(\bar{\Delta}_{2}\right)$.

При этом от функции $\nu_{2}$ требуется представление

$\nu_{2}(x, x+y)=\int_{x}^{h} T_{2}(\xi, x+y)\left[(\xi-x-y)^{2}-y^{2}\right]^{2 p}{ }_{0} F_{1}\left(1+p ;-\lambda\left[(\xi-x-y)^{2}-y^{2}\right]^{2}\right) d \xi$,

в котором $T_{2}(x, y)$ - непрерывная в $\Delta_{2}$ и интегрируемая в $\bar{\Delta}_{2}$ функция.

В выражении (15) функция $N_{2}$ определяется формулой

$$
N_{2}(\xi, s)=\frac{1}{2 \cos \pi p} T_{2}(\xi, s)+\frac{\Gamma(1+2 p)}{2 \Gamma^{2}(1+p)} \mu_{2}(\xi+s, s) .
$$

Непосредственными вычислениями доказывается, что функции (14), (15) (при выполнении предъявляемых к функциям $T_{i}, N_{i}, \tau_{i}, i \equiv 1,2$, требований) удовлетворяют уравнению $(1)$ в областях $\mathcal{G}_{1}$ и $\mathcal{G}_{2}$ соответственно, условиям задачи $\mathrm{C}$ и являются непрерывными в замкнутых областях.

2. Решение двумерных интегральных уравнений. Для решения задачи MS воспользуемся (14), (15), которые удовлетворяют условиям (2), (3). Неизвестные функции $N_{1}, N_{2}$ найдём из условий задачи (4), (5); полагая в (14), (15) $x=h$, получаем, с учётом требования (8), интегральные уравнения относительно $N_{i}(i \equiv 1,2)$ :

$$
\begin{array}{rl}
\varphi_{1}(y, z)=-\int_{z}^{h-y} & d s \int_{s+y}^{h} N_{1}(\xi, s)\left[(\xi-s)^{2}-y^{2}\right]^{p}\left[(h-s)^{2}-(\xi-s)^{2}\right]^{p} \times \\
& \times{ }_{0} F_{1}\left(1+p ; \lambda\left[(\xi-s)^{2}-y^{2}\right]\left[(h-s)^{2}-(\xi-s)^{2}\right]\right) d \xi
\end{array}
$$




$$
\begin{array}{rl}
\varphi_{2}(y, z)=-\int_{z}^{h+y} & d s \int_{s-y}^{h} N_{2}(\xi, s)\left[(\xi-s)^{2}-y^{2}\right]^{p}\left[(h-s)^{2}-(\xi-s)^{2}\right]^{p} \times \\
& \times{ }_{0} F_{1}\left(1+p ; \lambda\left[(\xi-s)^{2}-y^{2}\right]\left[(h-s)^{2}-(\xi-s)^{2}\right]\right) d \xi
\end{array}
$$

В уравнении (16) обозначим $N_{1}(\xi, s)\left[(h-s)^{2}-(\xi-s)^{2}\right]^{p} \equiv N(\xi, s)$. В предположении, что решение уравнения существует, продифференцируем обе его части сначала по $z$, затем по $y$, заменим $y$ на $t$ и применим к обеим частям полученного равенства оператор $\int_{y}^{h-z} \ldots\left(t^{2}-y^{2}\right)^{-p} d t$. В результате получим

$$
\begin{aligned}
\int_{y+z}^{h} N(\xi, z)_{0} F_{1}(1 ; \lambda[ & {\left.\left[(\xi-z)^{2}-y^{2}\right]\left[(h-z)^{2}-(\xi-z)^{2}\right]\right) d \xi=} \\
& =-\frac{1}{\Gamma(1+p) \Gamma(1-p)} \int_{y}^{h-z} \varphi_{1}^{\prime \prime}(t, z)\left(t^{2}-y^{2}\right)^{-p} d t
\end{aligned}
$$

В интеграле левой части (18) сделаем замену $\xi=z+t$, а затем обозначим $h-z=b$ :

$$
\int_{y}^{b} N(z+t, z)_{0} F_{1}\left(1 ; \lambda\left[t^{2}-y^{2}\right]\left[b^{2}-t^{2}\right]\right) d t=f(z, y)
$$

где $f(z, y)$ - известная функция, определённая формулой

$$
f(z, y)=-\frac{1}{\Gamma(1+p) \Gamma(1-p)} \int_{y}^{b} \varphi_{1}^{\prime \prime}(t, z)\left(t^{2}-y^{2}\right)^{-p} d t
$$

Продифференцируем обе части(19) по $y$ :

$$
-N(z+y, z)+\int_{y}^{b} N(z+t, z) \frac{\partial}{\partial y}{ }_{0} F_{1}\left(1 ; \lambda\left[t^{2}-y^{2}\right]\left[b^{2}-t^{2}\right]\right) d t=\frac{\partial}{\partial y} f(z, y)
$$

Далее, следуя идее работы [5], в формуле (21) заменим $y$ на $\tau$ и применим к обеим частям оператор

$$
\int_{x}^{b} \ldots\left(b^{2}-\tau^{2}\right) \tau^{-1} \frac{\partial}{\partial \tau}{ }_{0} F_{1}\left(1 ;-\lambda\left[\tau^{2}-x^{2}\right]\left[b^{2}-x^{2}\right]\right) d \tau .
$$

В полученном выражении обозначим второе слагаемое через $J(x, z)$ и поменяем в нём порядок интегрирования:

$$
J(x, z)=\int_{x}^{b} N(z+t, t) K(t, x) d t
$$

где 


$$
\begin{aligned}
K(t, x)=\int_{x}^{t}\left(b^{2}-\tau^{2}\right) \tau^{-1} \frac{\partial}{\partial \tau}{ }^{0} F_{1}\left(1 ;-\lambda\left[\tau^{2}\right.\right. & \left.\left.-x^{2}\right]\left[b^{2}-x^{2}\right]\right) \times \\
& \times \frac{\partial}{\partial \tau}{ }_{0} F_{1}\left(1 ; \lambda\left[t^{2}-\tau^{2}\right]\left[b^{2}-t^{2}\right]\right) d \tau .
\end{aligned}
$$

В интеграле $K(t, x)$ представим функции рядами, применим правило перемножения рядов, поменяем порядок суммирования и интегрирования и после вычисления приходим к

$$
\begin{aligned}
K(t, x)=\frac{b^{2}-t^{2}}{t} \frac{\partial}{\partial t}{ }_{0} F_{1}\left(1 ;-\lambda\left[t^{2}-x^{2}\right]\left[b^{2}-x^{2}\right]\right)- & \\
& -\frac{b^{2}-x^{2}}{x} \frac{\partial}{\partial x}{ }_{0} F_{1}\left(1 ; \lambda\left[t^{2}-x^{2}\right]\left[b^{2}-t^{2}\right]\right) .
\end{aligned}
$$

После применения к обеим частям (21) оператора (22), получим

$$
\begin{aligned}
-\int_{x}^{b}\left(b^{2}-x^{2}\right) x^{-1} N(z+t, z) \frac{\partial}{\partial x}{ }_{0} F_{1}\left(1 ; \lambda\left[b^{2}-t^{2}\right]\left[t^{2}-x^{2}\right]\right) d t= \\
=\int_{x}^{b} \frac{\partial}{\partial \tau} f(z, \tau) \tau^{-1}\left(b^{2}-\tau^{2}\right) \frac{\partial}{\partial \tau}{ }_{0} F_{1}\left(1 ;-\lambda\left[\tau^{2}-x^{2}\right]\left[b^{2}-x^{2}\right]\right) d \tau .
\end{aligned}
$$

Заменяя в (21) y на $x$, умножим обе части на $\left(b^{2}-x^{2}\right) x^{-1}$ и сложим полученное c (23):

$$
\begin{aligned}
-N(z+x, z)\left(b^{2}-x^{2}\right) x^{-1}= & \\
=\int_{x}^{b} \frac{\partial}{\partial \tau} f(z, \tau) \tau^{-1}\left(b^{2}-\tau^{2}\right) \frac{\partial}{\partial \tau}{ }_{0} F_{1}(1 ;-\lambda & {\left.\left[\tau^{2}-x^{2}\right]\left[b^{2}-x^{2}\right]\right) d \tau+} \\
& +\frac{\partial}{\partial x} f(z, x) x^{-1}\left(b^{2}-x^{2}\right) .
\end{aligned}
$$

После некоторых преобразований получим

$$
\begin{aligned}
& N_{1}(\xi, s)=\frac{\left[(h-s)^{2}-(\xi-s)^{2}\right]^{-p}}{\Gamma(1-p) \Gamma(1+p)}\left[(\xi-s) \int_{\xi-s}^{h-s}\left(\frac{\varphi_{1}^{\prime \prime}(t, z)}{t}\right)_{t}^{\prime} \times\right. \\
& \times\left[t^{2}-(\xi-s)^{2}\right]^{-p}{ }_{0} F_{1}\left(1-p ;-\lambda\left[t^{2}-(\xi-s)^{2}\right]\left[(h-s)^{2}-(\xi-s)^{2}\right]\right) d t+ \\
& \quad+\frac{2 \lambda}{1-p} \int_{\xi-s}^{h-s} \varphi_{1 s t}^{\prime \prime}(t, s)\left[t^{2}-(\xi-s)^{2}\right]^{1-p} \times \\
& \left.\times{ }_{0} F_{1}\left(2-p ;-\lambda\left[t^{2}-(\xi-s)^{2}\right]\left[(h-s)^{2}-(\xi-s)^{2}\right]\right) d t\right]
\end{aligned}
$$

Подобными рассуждениями получим решение уравнения (17):

$$
N_{2}(\xi, s)=-\frac{\left[(h-s)^{2}-(\xi-s)^{2}\right]^{-p}}{\Gamma(1-p) \Gamma(1+p)}\left[(\xi-s) \int_{\xi-s}^{h-s}\left(\frac{\varphi_{2}^{\prime \prime}(-t, s)}{t}\right)_{t}^{\prime} \times\right.
$$




$$
\begin{gathered}
\times\left[t^{2}-(\xi-s)^{2}\right]_{0}^{-p} F_{1}\left(1-p ;-\lambda\left[t^{2}-(\xi-s)^{2}\right]\left[(h-s)^{2}-(\xi-s)^{2}\right]\right) d t+ \\
+\frac{2 \lambda}{1-p} \int_{\xi-s}^{h-s} \varphi_{2}^{\prime \prime}{ }_{s t}^{\prime \prime}(-t, s)\left[t^{2}-(\xi-s)^{2}\right]^{1-p} \times \\
\left.\quad \times{ }_{0} F_{1}\left(2-p ;-\lambda\left[t^{2}-(\xi-s)^{2}\right]\left[(h-s)^{2}-(\xi-s)^{2}\right]\right) d t\right] .
\end{gathered}
$$

Функции (25), (26) при выполнении условий (10)-(13) непрерывны в $\bar{\Delta}_{1}$.

Непрерывность решения задачи MS на $y=0$ и условия сопряжения (6) приводит к интегральным уравнениям относительно $T_{1} \pm T_{2}$ :

$$
\begin{gathered}
\int_{z}^{x} d s \int_{x}^{h}\left[T_{1}(\xi, s)-T_{2}(\xi, s)\right](\xi-s)^{2 p}\left[(\xi-s)^{2}-(x-s)^{2}\right]^{p} \times \\
\times{ }_{0} F_{1}\left(1+p ;-\lambda[\xi-s]^{2}\left[(\xi-s)^{2}-(x-s)^{2}\right]\right) d \xi= \\
=\int_{z}^{x} d s \int_{s}^{x}\left[N_{2}(\xi, s)-N_{1}(\xi, s)\right](\xi-s)^{2 p}\left[(x-s)^{2}-(\xi-s)^{2}\right]^{p} \times \\
\times{ }_{0} F_{1}\left(1+p ; \lambda[\xi-s]^{2}\left[(x-s)^{2}-(\xi-s)^{2}\right]\right) d \xi \\
\int_{z}^{x}(x-s) d s \int_{x}^{h}\left[T_{1}(\xi, s)+T_{2}(\xi, s)\right](\xi-s)^{2 p}\left[(\xi-s)^{2}-(x-s)^{2}\right]^{p-1} \times \\
\times{ }_{0} F_{1}\left(p ;-\lambda[\xi-s]^{2}\left[(\xi-s)^{2}-(x-s)^{2}\right]\right) d \xi= \\
=\int_{z}^{x}(x-s) d s \int_{s}^{x}\left[N_{2}(\xi, s)+N_{1}(\xi, s)\right](\xi-s)^{2 p}\left[(x-s)^{2}-(\xi-s)^{2}\right]^{p-1} \times \\
\times{ }_{0} F_{1}\left(p ; \lambda[\xi-s]^{2}\left[(x-s)^{2}-(\xi-s)^{2}\right]\right) d \xi
\end{gathered}
$$

при выполнении условий (8), (9).

Применяя такой же метод как и при решении уравнения (16), получим

$$
\begin{gathered}
T_{1}(\xi, z)=\frac{(\xi-z)^{1-2 p}}{\Gamma(p) \Gamma(1-p)}\left[\frac{\lambda}{1-p} \int_{\xi-z}^{h-z}\left[\Psi_{1}(t, z)+\Psi_{2}(t, z)\right]\left(t^{2}-(\xi-z)^{2}\right)^{1-p} \times\right. \\
\times{ }_{0} F_{1}\left(2-p ; \lambda[\xi-z]^{2}\left[t^{2}-(\xi-z)^{2}\right]\right) d\left(t^{2}\right)- \\
-\int_{\xi-z}^{h-z}\left[\Psi_{1}(t, z)+\Psi_{2}(t, z)\right]_{t}^{\prime}\left(t^{2}-(\xi-z)^{2}\right)^{-p} \times \\
\left.\times{ }_{0} F_{1}\left(1-p ; \lambda[\xi-z]^{2}\left[t^{2}-(\xi-z)^{2}\right]\right) d t\right], \\
T_{2}(\xi, z)=\frac{(\xi-z)^{1-2 p}}{\Gamma(p) \Gamma(1-p)}\left[\frac{\lambda}{1-p} \int_{\xi-z}^{h-z}\left[\Psi_{1}(t, z)-\Psi_{2}(t, z)\right]\left(t^{2}-(\xi-z)^{2}\right)^{1-p} \times\right. \\
\times{ }_{0} F_{1}\left(2-p ; \lambda[\xi-z]^{2}\left[t^{2}-(\xi-z)^{2}\right]\right) d\left(t^{2}\right)-
\end{gathered}
$$




$$
\begin{aligned}
-\int_{\xi-z}^{h-z}\left[\Psi_{1}(t, z)\right. & \left.-\Psi_{2}(t, z)\right]_{t}^{\prime}\left(t^{2}-(\xi-z)^{2}\right)^{-p} \times \\
& \left.\times{ }_{0} F_{1}\left(1-p ; \lambda[\xi-z]^{2}\left[t^{2}-(\xi-z)^{2}\right]\right) d t\right]
\end{aligned}
$$

где

$$
\begin{aligned}
& \Psi_{1}(t, z) \equiv \int_{z}^{t+z}\left[N_{1}(\xi, z)+N_{2}(\xi, z)\right](\xi-z)^{2 p}\left[t^{2}-(\xi-z)^{2}\right]^{p-1} \times \\
& \times{ }_{0} F_{1}\left(p ; \lambda[\xi-z]^{2}\left[t^{2}-(\xi-z)^{2}\right]\right) d \xi \\
& \Psi_{2}(t, z) \equiv-\int_{z}^{t+z}\left[N_{1}(\xi, z)-N_{2}(\xi, z)\right](\xi-z)^{2 p}\left[t^{2}-(\xi-z)^{2}\right]^{p-1} \times \\
& \times{ }_{0} F_{1}\left(p ; \lambda[\xi-z]^{2}\left[t^{2}-(\xi-z)^{2}\right]\right) d \xi
\end{aligned}
$$

Проверка показала, что для разрешимости уравнений (27), (28) нужно наложить дополнительное требование на заданные функции $\varphi_{i}$ :

$$
\frac{\partial \varphi_{1}(0, z)}{\partial z}=\frac{\partial \varphi_{2}(0, z)}{\partial z}=0
$$

Легко показать, что при выполнении условий (7)-(13) функции $T_{i}(x, y)$ непрерывны в соответствующих областях $\Delta_{i}$ и интегрируемы в $\bar{\Delta}_{i}(i \equiv 1,2)$.

ТеОРема. При выполнении условий (7)-(13), (33) задача MS для уравнения (1) имеет единственное решение, определяемое формулами (14), (15), (25), (26), (29)-(32).

Единственность решения задачи следует из единственности решения видоизмененной задачи Коши, полученного методом Римана и взятого за основу при определении специального класса решений $R_{h}$, а также из однозначной разрешимости всех интегральных уравнений, участвующих в процессе решения.

Работа выполнена при финансовой поддержке Федерального агентства по образованию (проект АВЦП 3341).

\section{БИБЛИОГРАФИЧЕСКИЙ СПИСОК}

1. Долгополов В. М., Долгополов М. В., Родионова И. Н. Построение специальных классов решений некоторых дифференциальных уравнений гиперболического типа // ДАН. Maтематика, 2009. - Т. 429, № 5. - C. 583-589; англ. пер.: Dolgopolov V.M., Dolgopolov M. V., Rodionova I. N. Construction of special classes of solutions for some differential equations of hyperbolic type// Dokl. Math., 2009. - Vol. 80, No. 3. — P. 860-866.

2. Долгополов В. М., Родионова И. Н. Видоизмененная задача Коши для одного гиперболического уравнения третьего порядка в трёхмерном пространстве // Becmн. Caм. гос. техн. ун-та. Сер. Физ.-мат. науки, 2009. - № 1(18). - С. 41-46.

3. Долгополов В. М., Долгополов М. В., Родионова И. Н. Две задачи для гиперболического уравнения в трёхмерном пространстве // Вестн. Сам. гос. ун-та. Естественнонаучная сер., 2008. - № 8/1(67). - C. 95-102. 
4. Волкодавов В.Ф., Родионова И.Н., Бушков С.В. Пространственный аналог задачи $M$ для одного гиперболического уравнения третьего порядка// Дифферени. уравнения, 2004. - T. 40, №12. - C. 1698-1700; англ. пер.: Volkodavov V. F., Rodionova I. N., Bushkov S. V. A 3D analog of problem $M$ for a third-order hyperbolic equation// Differ. Equ., 2004. - Vol. 40, No.12. - P. 1781-1784.

5. Самко С. Г., Килбас А.А., Маричев О.И. Интегралы и производные дробного порядка и некоторые их приложения. - Мн.: Наука и техника, 1987. - 688 с.

Поступила в редакцию 15/I/2010;

в окончательном варианте - 22/III/2010.

MSC: 35G30, 35Q05, 35L25

\section{PROBLEM WITH CONJUGATION ON THE CHARACTERISTIC PLANE FOR ONE 3D SPACE ANALOGUE OF HYPERBOLIC TYPE EQUATION}

\section{M. Dolgopolov, I. N. Rodionova}

Samara State University,

1, Pavlova str., Samara, 443011

E-mail: mvdolg@ssu.samara.ru

The value boundary problem with conjugation together on the characteristic plane in the special class, entered by authors, is solved for the one 3D space analogue of the hyperbolic type equation in the area, which parts of border are the planes of singularity of the given equation factors.

Key words: integral equations, boundary value problems, hyperbolic type equations.

Original article submitted 15/I/2010; revision submitted $22 / \mathrm{III} / 2010$.

Vyacheslav M. Dolgopolov (Ph. D. (Phys. \& Math.)), Associate Professor, Dept. of Mathematics, Computer Science \& Mathematical Methods in Economy. Irina N. Rodionova (Ph. D. (Phys. \& Math.)), Associate Professor, Dept. of Mathematics, Computer Science \& Mathematical Methods in Economy. 